\title{
Stroke in Dialysis and Chronic Kidney Disease
}

\author{
Albert Power \\ Imperial College London, London, UK
}

\section{Key Words}

Chronic kidney disease $\cdot$ Dialysis · Stroke · Thrombolysis

\begin{abstract}
Background: Renal impairment is a potent risk factor for stroke that is a leading cause of morbidity and mortality worldwide. Dialysis patients experience a 10 -fold higher incidence, with case fatality rates reaching $90 \%$. It is important to understand the factors predisposing to stroke in patients with chronic kidney disease (CKD) coupled with an appreciation of preventative strategies. Summary: The heightened risk of stroke in CKD represents the interplay of the vascular comorbidities that cluster with renal impairment as well as pathology inherent in uremia, such as accelerated vascular calcification and the malnutrition-inflammation-atherosclerosis syndrome. These factors are most marked in hemodialysis where stroke rates peak at 10-35/1,000 patient years and where hemorrhagic stroke accounts for $20-30 \%$ of all events. Older age, hypertension, diabetes and established cerebrovascular disease are all risk factors for stroke with dialysis initiation constituting the highest risk period. Patients with CKD stages 3-5D have worse survival as well as diminished functional outcomes following stroke. Thrombolytic therapy for acute stroke appears safe in all stages of CKD al-
\end{abstract}

though the therapeutic effect may be attenuated. Control of hypertension and the use of antiplatelet agents form the mainstay of stroke prevention. The benefit of antiplatelet therapies and oral anticoagulants must be balanced against the real risks of bleeding that are most evident in dialysis cohorts. Key Messages: Understanding the risks and benefits of established stroke treatments is vital in patients with CKD, especially in those on dialysis therapies who are at highest risk of adverse outcomes.

(c) 2013 S. Karger AG, Basel

\section{Introduction}

Stroke remains a significant global health issue and is the leading cause of morbidity and mortality worldwide. In contrast to the declining stroke incidence in high-income countries, stroke rates continue to increase in low and middle-income nations [1]. Risk factors for stroke closely mirror those for cardiac and peripheral vascular disease. Non-modifiable risk factors include older age, diabetes, a positive family history, male gender and nonCaucasian ethnicity. Hypertension remains the major modifiable risk factor for both ischemic and hemorrhagic strokes with risk increasing in proportion to both sys-

\section{KARGER}

E-Mail karger@karger.com

www.karger.com/bpu
(C) 2013 S. Karger AG, Basel

0253-5068/13/0364-0179\$38.00/0
Dr. Albert Power

Room 5N8A, Commonwealth Building

Imperial College London, Hammersmith Hospital Campus

DuCane Road, London W12 0NN (UK)

E-Mail albert.power@nhs.net 
tolic and diastolic blood pressure (BP). Atherosclerotic risk factors such as smoking, diabetes mellitus and hyperlipidemia (particularly in people aged $\leq 45$ years) as well as atrial fibrillation (AF) confer a greater risk of ischemic stroke whereas high alcohol use, a bleeding diathesis and blood vessel wall fragility (e.g. as a result of congenital aneurysm or amyloid angiopathy, as seen typically in the elderly) predispose to hemorrhagic stroke [2]. Chronic kidney disease (CKD) is associated with an overrepresentation of traditional cardiovascular risk factors and an increased risk of stroke. Management of hypertension becomes an increasingly significant issue as renal function declines with a progressively adverse impact on stroke risk. Furthermore the prevalence of AF in CKD populations is over twice that in the general population and confers a greater thromboembolic risk [3]. Stroke rates increase with declining renal function and reach a zenith in patients with end-stage renal disease (ESRD).

\section{Stroke Risk in CKD}

The clustering of vascular risk factors in patients with CKD has been suggested as the salient reason for the observed association of renal dysfunction with stroke. However, some studies reported a graded and independent relationship between estimated glomerular filtration rate (eGFR) and stroke risk. A recent meta-analysis incorporating data from 33 studies reported a $43 \%$ independently greater risk of stroke associated with eGFR $<60 \mathrm{ml} / \mathrm{min}$ [4]. This effect was further modulated by ethnicity, with a higher stroke risk seen in Asian compared to non-Asian populations (relative risk 1.96 vs. $1.26, \mathrm{p}<0.001$ ). Proteinuria is itself an important risk factor for stroke even in the absence of reduced eGFR and after adjusting for other vascular risk factors (risk ratio 1.71, 95\% CI 1.392.10, $\mathrm{p}=0.008)$ [5].

The pathophysiological mechanisms mediating an elevated stroke risk in CKD are unclear but may relate to factors specific to the uremic milieu, such as accelerated vascular calcification, increased carotid atherosclerosis, a prothrombotic tendency and impaired cerebral autoregulation. Intracranial arterial calcification, for example, is associated with stroke risk in the general population and increases in prevalence in patients with CKD presenting with stroke-like symptoms. However, a study in hemodialysis (HD) patients which reported a high prevalence of intracranial arterial calcification in HD patients did not find an independent association with acute stroke ( $\mathrm{p}=$ 0.08) [6].

\section{Stroke Risk in Dialysis}

Patients with ESRD on dialysis have an 8-10 times greater incidence of stroke compared to the general population, with rates varying across published series from 10 to 33 per 1,000 patient years $[7,8]$. Furthermore, there is a higher prevalence of hemorrhagic strokes compared to the general population ( $20 \%$ all events), a finding which was particularly marked in early Japanese studies that reported hemorrhagic stroke in up to $80 \%$ cases. This may relate to the degree of hypervolemia and hypertension seen in HD patients as well as the regular use of anticoagulation to maintain patency of the extracorporeal circuit.

It is important to note that the overwhelming majority of data in dialysis patients derives from HD cohorts centered on the US and Japan. In contrast, rates of stroke in peritoneal dialysis patients are less well characterized. In the largest peritoneal dialysis cohort to date from the United Kingdom ( $\mathrm{n}=1,511$ with a mean age of 55 years), the overall incidence of stroke was 9.8 per 1,000 patient years [9]. US registry studies reported no differences in stroke risk in patients on peritoneal dialysis compared to those on HD [10].

The initiation of dialysis itself is associated with a heightened risk of stroke. In an analysis of just under 21,000 US dialysis patients aged $\geq 67$ years, stroke rates began to rise about 3 months before dialysis initiation and reached a peak during the first 30 days of dialysis. This pattern was preserved irrespective of dialysis modality and whether the patients started dialysis in a planned manner [10]. Emerging data from the international MONDO dialysis research initiative suggest that similar temporal variations in stroke incidence occur also in younger cohorts and that this finding constitutes a global phenomenon [unpubl. data]. In one Japanese study, 39\% of ischemic and $35 \%$ of hemorrhagic strokes occurred during or within $30 \mathrm{~min}$ of concluding $\mathrm{HD}$, suggesting that the treatment itself may mediate stroke risk [11]. More studies are required to investigate this further and determine treatment parameters that constitute a cerebral vascular insult. Additional risk factors associated with stroke in ESRD patients include older age, hypertension, low serum albumin, diabetes mellitus and established cerebrovascular disease (i.e. prior stroke or transient ischemic attack, TIA).

The prevalence of AF in HD populations is up to 10 times greater than that in the general population. There have been conflicting data regarding the role of AF as a stroke risk factor in these cohorts. In the largest epide- 
miological study to date, chronic AF was independently associated with a modest risk of ischemic stroke (hazard ratio $1.26, \mathrm{p}<0.001)$ [12].

\section{Stroke Outcomes in CKD and Dialysis}

$\mathrm{CKD}$ is an independent risk factor for both ischemic as well as hemorrhagic stroke. In addition, renal impairment is associated with a greater neurological deficit following ischemic stroke, a poor functional outcome and greater mortality irrespective of the stroke subtype [13]. Following acute ischemic stroke, advanced CKD (eGFR $<30 \mathrm{ml} / \mathrm{min}$ ) has been associated with a higher risk of hemorrhagic transformation (odds ratio 2.90, 95\% CI $1.26-6.68, p=0.01)$ [14]. Furthermore, in patients with hemorrhagic stroke, moderate-to-severe CKD has been associated with a 2.3 times greater hematoma volume [15]. Taken together, these findings may represent the older age and comorbid profile of patients with CKD who experience a stroke or reflect the preexisting, subclinical cerebral vascular disease burden seen in patients with advanced renal impairment.

Systemic thrombolytic therapy with recombinant tissue plasminogen activator (alteplase) is the standard of care for patients presenting within $4.5 \mathrm{~h}$ of symptom onset following acute ischemic stroke. Results from IST-3 (the Third International Stroke Trial) suggest that this timeframe can safely be extended to $6 \mathrm{~h}$ and include older patients that were excluded from previous large trials. However, all these randomized controlled trials (RCTs) did not capture renal function, so the safety and efficacy of this approach in patients with CKD and ESRD is unclear. Analysis of available retrospective series is confounded by different study populations, varying definitions of CKD, alteplase dosing protocols and end points [16-18]. Overall, the data suggest that CKD attenuates the therapeutic efficacy of alteplase, and in one study increased the risks of symptomatic intracranial hemorrhage after treatment. In the only available large, registrybased study of US dialysis patients receiving thrombolysis for stroke $(n=1,042)$, there was no difference in the rates of symptomatic intracranial hemorrhage or disability at discharge although dialysis patients had a 2 -fold higher risk of inhospital mortality following thrombolysis [19].

Acute stroke constitutes a highly significant clinical event for patients with renal impairment who have an overall greater risk of inhospital death (odds ratio 1.63, $95 \%$ CI $1.52-1.75, \mathrm{p}<0.001$ ), a relationship which becomes more marked with advancing CKD stage [20].
Similarly, HD patients have a 3-fold higher risk of death following acute stroke, which is independent of traditional risk factors $[8,20]$. The prognosis following hemorrhagic stroke is particularly poor with case fatality rates reaching $90 \%$ in some series. It remains unclear whether ESRD patients have worse functional outcomes and quality of life following acute stroke.

\section{Stroke Prevention in CKD and Dialysis}

Traditionally, patients with advanced renal impairment have been excluded from RCTs examining the impact of healthcare interventions on the occurrence of stroke. As a result, treatment paradigms from the general population have been applied in patients with all forms of renal impairment. Recent RCTs in dialysis cohorts have indicated that such presumptions of efficacy may be misleading. Control of hypertension is the cornerstone of primary and secondary stroke prevention in the general population as well as in patients with nondialysis CKD. The relationship between attained BP and stroke risk was shown to be linear in a recent RCT although prior, large, epidemiological studies suggested that in patients with CKD 3-4 this relationship was Jshaped with a systolic BP $<120 \mathrm{~mm} \mathrm{Hg}$ associated with a 2.5 times greater risk $[21,22]$. To date, there are no compelling trial data to recommend one class of antihypertensives over another. Although retrospective studies have indicated that uncontrolled hypertension in dialysis patients is associated with stroke there are no studies defining an optimal target BP.

Clinical guidelines advocate the use of antiplatelet therapy for the prevention of ischemic stroke and there is evidence supporting its efficacy in patients with non-dialysis CKD [23]. The risk of bleeding with antiplatelet agents is augmented in ESRD and caution is advised. Stroke thromboprophylaxis with oral anticoagulants (warfarin and, more recently, with newer agents such as dabigatran or rivaroxaban) is recommended in patients with AF. Once more, there are no randomized controlled trials in CKD or ESRD patients, but efficacy in non-dialysis CKD is supported by data from a Danish study [24]. Treatment with oral anticoagulants needs to be tempered by the higher risk of bleeding seen in patients with renal impairment and especially in those with ESRD [25]. Paradoxically, warfarin use has been associated with an increased risk of stroke in HD patients possibly due to accelerated vascular calcification occurring as a result of vitamin K antagonism [26]. 
SHARP (the Study of Heart and Renal Protection) demonstrated that lipid lowering with statin therapy is effective at reducing the risk of stroke in patients with CKD 3-4 [27]. It is important to note that in a subgroup of dialysis patients enrolled in SHARP as well as other RCTs in dialysis patients, statin therapy did not have a significant effect in reducing stroke risk $[27,28]$. The rationale supporting the use of these agents in ESRD remains contentious.

Rapid assessment and risk factor modification in patients presenting with TIA have been shown to reduce the incidence of subsequent acute stroke. The relationship between TIA and acute stroke in patients with advanced renal impairment is unclear and the impact of such a multifactorial intervention in ESRD has not been studied. In a study from the United Kingdom, systematic symptom screening for TIA in HD patients did not result in better ascertainment of this syndrome nor did it identify patients who subsequently had an acute stroke [29]. This suggests that the cardinal relationship between TIA and stroke may not be as robust in HD patients or that diagnosis itself may be obfuscated by the significant symptom burden of this cohort.

Carotid endarterectomy is recommended for patients with symptomatic, high-grade $(>70 \%)$ carotid stenosis to reduce subsequent stroke risk. Endarterectomy reduced the risk of stroke by $82 \%$ at 2 years in patients with stage 3 CKD [30]. The number needed to treat was 4 in patients with CKD compared to 10 for patients with preserved
eGFR. In this study, patients with CKD had similar rates of perioperative mortality but higher rates of cardiac events. Similar data in HD patients are scant but suggest that dialysis patients are at no increased risk of perioperative events although the long-term benefits of this procedure are less clear.

\section{Conclusion}

Stroke is a devastating expression of vascular risk in $\mathrm{CKD}$ and yet, in comparison to cardiac disease, is relatively understudied. It is vital to understand the risks as well as the benefits of established treatments directed at stroke treatment and prevention in all stages of CKD. Elements of the dialysis treatment itself, particularly in patients on HD, may mediate stroke risk as well as influence recovery from acute stroke.

\section{Acknowledgment}

Dr. Power is supported by the UK National Institute for Health Research and funding from the Imperial College Biomedical Research Council.

\section{Disclosure Statement}

No conflicts of interest to declare.

\section{References}

1 Norrving B, Kissela B: The global burden of stroke and need for a continuum of care. Neurology 2013;80(3 suppl 2):S5-S12.

2 O’Donnell MJ, Xavier D, Liu L, Zhang H, Chin SL, Rao-Melacini P, et al: Risk factors for ischaemic and intracerebral haemorrhagic stroke in 22 countries (the INTERSTROKE study): a case-control study. Lancet 2010;376: 112-123.

- 3 Go AS, Fang MC, Udaltsova N, Chang Y, Pomernacki NK, Borowsky L, et al: Impact of proteinuria and glomerular filtration rate on risk of thromboembolism in atrial fibrillation: the anticoagulation and risk factors in atrial fibrillation (ATRIA) study. Circulation 2009; 119:1363-1369.

4 Lee M, Saver JL, Chang KH, Liao HW, Chang SC, Ovbiagele B: Low glomerular filtration rate and risk of stroke: meta-analysis. BMJ 2010;341:c4249.
5 Ninomiya T, Perkovic V, Verdon C, Barzi F, Cass A, Gallagher M, et al: Proteinuria and stroke: a meta-analysis of cohort studies. Am J Kidney Dis 2009;53:417-425.

-6 Power A, Chan K, Haydar A, Hamady M, Cairns T, Taube D, et al: Intracranial arterial calcification is highly prevalent in hemodialysis patients but does not associate with acute ischemic stroke. Hemodial Int 2011;15:256-263.

7 Foley RN, Gilbertson DT, Murray T, Collins AJ: Long interdialytic interval and mortality among patients receiving hemodialysis. N Engl J Med 2011;365:1099-1107.

8 Power A, Chan K, Singh SK, Taube D, Duncan N: Appraising stroke risk in maintenance hemodialysis patients: a large single-center cohort study. Am J Kidney Dis 2012;59:249257.

9 Power A, Davenport A, Brown EA, Duncan N, Fan S: Stroke incidence and risk factors in peritoneal dialysis (abstract). J Am Soc Nephrol 2012;23:227A.
10 Murray AM, Seliger S, Lakshminarayan K, Herzog CA, Solid CA: Incidence of stroke before and after dialysis initiation in older patients. J Am Soc Nephrol 2013;24:1166-1173.

11 Toyoda K, Fujii K, Fujimi S, Kumai Y, Tsuchimochi $\mathrm{H}$, Ibayashi S, et al: Stroke in patients on maintenance hemodialysis: a 22-year single-center study. Am J Kidney Dis 2005;45: 1058-1066.

12 Wetmore JB, Ellerbeck EF, Mahnken JD, Phadnis M, Rigler SK, Mukhopadhyay P, et al: Atrial fibrillation and risk of stroke in dialysis patients. Ann Epidemiol 2013;23:112-118.

13 Ovbiagele B, Sanossian N, Liebeskind DS, Kim D, Ali LK, Pineda S, et al: Indices of kidney dysfunction and discharge outcomes in hospitalized stroke patients without known renal disease. Cerebrovasc Dis 2009;28:582588. 
14 Lee JG, Lee KB, Jang IM, Roh H, Ahn MY, Woo HY, et al: Low glomerular filtration rate increases hemorrhagic transformation in acute ischemic stroke. Cerebrovasc Dis 2013; 35:53-59.

15 Molshatzki N, Orion D, Tsabari R, Schwammenthal Y, Merzeliak O, Toashi M, et al: Chronic kidney disease in patients with acute intracerebral hemorrhage: association with large hematoma volume and poor outcome. Cerebrovasc Dis 2011;31:271-277.

16 Agrawal V, Rai B, Fellows J, McCullough PA: In-hospital outcomes with thrombolytic therapy in patients with renal dysfunction presenting with acute ischaemic stroke. Nephrol Dial Transplant 2010;25:1150-1157.

-17 Naganuma M, Koga M, Shiokawa Y, Nakagawara J, Furui E, Kimura K, et al: Reduced estimated glomerular filtration rate is associated with stroke outcome after intravenous rtPA: the Stroke Acute Management with Urgent Risk-Factor Assessment and Improvement (SAMURAI) rt-PA registry. Cerebrovasc Dis 2011;31:123-129.

18 Power A, Epstein D, Cohen D, Bathula R, Devine J, Kar A, et al: Renal impairment reduces the efficacy of thrombolytic therapy in acute ischemic stroke. Cerebrovasc Dis 2013; 35:45-52.
19 Tariq N, Adil MM, Saeed F, Chaudhry SA, Qureshi AI: Outcomes of thrombolytic treatment for acute ischemic stroke in dialysis-dependent patients in the United States. J Stroke Cerebrovasc Dis 2013, E-pub ahead of print.

20 Ovbiagele B: Chronic kidney disease and risk of death during hospitalization for stroke. Neurol Sci 2011;301:46-50.

21 Ninomiya T, Perkovic V, Gallagher M, Jardine M, Cass A, Arima H, et al: Lower blood pressure and risk of recurrent stroke in patients with chronic kidney disease: Progress trial. Kidney Int 2008;73:963-970.

22 Weiner DE, Tighiouart H, Levey AS, Elsayed E, Griffith JL, Salem DN, et al: Lowest systolic blood pressure is associated with stroke in stages 3-4 chronic kidney disease. J Am Soc Nephrol 2007;18:960-966.

23 Jardine MJ, Ninomiya T, Perkovic V, Cass A, Turnbull F, Gallagher MP, et al: Aspirin is beneficial in hypertensive patients with chronic kidney disease: a post-hoc subgroup analysis of a randomized controlled trial. Am Coll Cardiol 2010;56:956-965.

24 Olesen JB, Lip GY, Kamper AL, Hommel K, Kober L, Lane DA, et al: Stroke and bleeding in atrial fibrillation with chronic kidney disease. N Engl J Med 2012;367:625-635.
25 Sood MM, Larkina M, Thumma JR, Tentori F, Gillespie BW, Fukuhara S, et al: Major bleeding events and risk stratification of antithrombotic agents in hemodialysis: results from the DOPPS. Kidney Int 2013;84:600608.

26 Chan KE, Lazarus JM, Thadhani R, Hakim RM: Warfarin use associates with increased risk for stroke in hemodialysis patients with atrial fibrillation. J Am Soc Nephrol 2009;20: 2223-2233.

27 Baigent C, Landray MJ, Reith C, Emberson J, Wheeler DC, Tomson C, et al: The effects of lowering LDL cholesterol with simvastatin plus ezetimibe in patients with chronic kidney disease (Study of Heart and Renal Protection): a randomised placebo-controlled trial. Lancet 2011;377:2181-2192.

-28 Wanner C, Krane V, Marz W, Olschewski M, Mann JF, Ruf G, et al: Atorvastatin in patients with type 2 diabetes mellitus undergoing hemodialysis. N Engl J Med 2005;353:238-248.

29 Power A, Edwards C, Sawyer J, Tandaric D, Dahdaleh D, Taube D, et al: Screening for transient ischemic attacks in hemodialysis patients. J Nephrol 2013;26:919-924.

30 Mathew A, Eliasziw M, Devereaux PJ, Merino JG, Barnett HJ, Garg AX, et al: Carotid endarterectomy benefits patients with CKD and symptomatic high-grade stenosis. J Am Soc Nephrol 2010;21:145-152. 